\title{
Development and Standardization of Testing Equipment and Methods for Spacer Fabrics
}

\author{
Carolin Schwager $^{1} \cdot$ Christoph Peiner $^{1} \cdot$ Isa Bettermann ${ }^{1} \cdot$ Thomas Gries $^{1}$
}

Received: 12 May 2021 / Accepted: 9 August 2021 / Published online: 20 January 2022

(c) The Author(s) 2021

\begin{abstract}
Spacer fabrics are three-dimensional textile structures consisting of two textile cover surfaces and a spacer thread. Up to now, spacer fabrics have been tested according to standards for flat textiles or other non-textile materials. However, these standards do not take into account the special requirements for testing spacer fabrics. Some sample holders of the testing devices are not designed for the thickness of the spacer fabrics, so that the tests cannot be carried out. In other tests, the samples can be mounted, but the test results are falsified by the methodology, e.g. strong compression of the sample before testing. Therefore, objective comparisons among spacer fabrics or between spacer fabrics and conventional flat textiles or non-textile materials cannot be made in all areas of application. The focus of this paper is in the developing of testing devices for seven test setups (1. sample preparation, 2. maximum force, 3. thickness, 4. compression, 5. mass per unit area, 6. permeability to air, 7. abrasion resistance). The new testing devices and methods were designed and manufactured using the method of an iterative development process. The following steps were carried out identically for all seven test setups: deficit analysis, development of concepts, construction of test benches, evaluation, transfer into standards. As part of this research work the developed devices where both tested and evaluated by industrial partners as well as later translated into a standard by the German Institute for Standardization (DIN e.V.). As a central result, a first standard for the testing of spacer fabrics was created and published: DIN 60022-1 "Spacer textiles - Terms and definitions, sample preparation" [4]. For testing textiles, it is important that geometrically identical and structurally intact samples are prepared. Therefore, this standard provides measurement tools and methods for the evaluation of sample quality (e.g. roundness of circular samples, maximum offset and shearing of the surfaces). Two further test methods (determination of thickness and air permeability) were developed and are now being transferred to standards. Within the new test standards, the special properties of spacer fabrics are given special consideration. In addition to the test methods developed within this work, further research is necessary. In particular, the tilting stability (linked to the in-plane and out-of-plane shear measurement) as well as the compression behaviour of spacer fabrics are important tests that need to be analysed and further developed. Therefore, further research is planned for six test methods (1. compression hardness, 2. compression set, 3. tilting stability, 4. pressure point distribution, 5. abrasion resistance, 6. maximum force). This work enables standardized testing
\end{abstract}


of spacer fabrics and thus objective comparisons not only between various spacer fabric constructions but also with conventional flat textiles and with non-textile materials.

Keywords Spacer fabric $\cdot$ Testing $\cdot$ Standardization $\cdot$ Sample preparation $\cdot$ Air permeability $\cdot$ Thickness

\section{Introduction}

Spacer fabrics are textile structures consisting of two textile cover surfaces and at least one spacer thread system. The cover surfaces are kept at a distance by the spacer thread (Fig. 1). Due to this structure, spacer fabrics have special properties that make them a substitute material for other, non-textile materials (e.g. foams). These are, among others, the following properties:

- Damping

- Air permeability

- Functionalisation due to different surfaces textures

- Design due to different surfaces textures

- Different pore sizes

- Stabilisation layer, keeping both surfaces at a distance

- Thickness

- Stiffness

Spacer fabrics can be made as woven, warp knitted or weft knitted fabrics. Applications can be found wherever increased air circulation, spacing between cover surfaces or a comfort effect due to the damping structure is desired. Warp knitted spacer fabrics, for example, are used in automotive interiors as a climate comfort layer for improved air circulation in the seat. Annual production is estimated at $60,000 \mathrm{~m}^{2}$ per year. Weft knitted spacer fabrics, on the other hand, are frequently used in mattress cover fabrics. Annual sales in the sector of mattress cover fabrics are estimated at just under $€ 20$ million [1].

Because of their structure, the properties of spacer fabrics differ from conventional flat textiles. Though, up to now, spacer fabrics have been tested according to standards for flat textiles or other, non-textile materials. These standards do not take into account the special

Fig. 1 Warp knitted spacer fabric

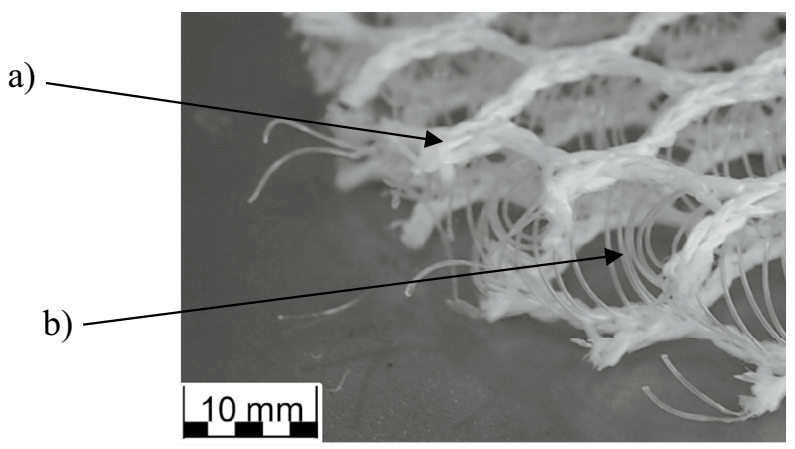

a) Textile cover surface, b) Spacer thread 
requirements of testing spacer fabrics. Therefore, objective comparisons of spacer fabrics with other spacer fabrics or with conventional textiles or non-textile materials cannot be made in all areas of application.

Consequently, it is necessary to develop new test benches and test methods allowing the consideration of the special properties of spacer fabrics during testing. This will allow the standardized testing of these properties and thus objective comparisons between.

- spacer fabrics,

- spacer fabrics and conventional, flat textiles, and

- spacer fabrics and non-textile materials.

In order to develop suitable test methods, the existing standards will be shown and evaluated. The focus of this paper is in the developing of testing devices for seven test-set ups:

1. Sample preparation

2. Maximum force

3. Thickness

4. Compression

5. Mass per unit area

6. Permeability to air

7. Abrasion resistance

\section{State of the Art Deficits of Available Testing Methods of 2D Textiles for Spacer Fabrics}

In the following chapter, the available test methods for 2D textiles are described and their deficits for spacer fabrics are identified. The following table is a summary overview of important textile testing standards and lists the respective deficits for the testing of spacer fabrics (Table 1).

\subsection{Research Strategy}

Within this work the method of an iterative development process is applied. First, various spacer fabrics are tested using conventional test methods. The causes of faults in the testing of spacer fabrics (woven, warp knitted and weft knitted) are analyzed. The development of the test methods is done in parallel within five phases for each test setup (Fig. 2):

1. analysis of the deficits of existing standards for 2D textiles with regard to their application for 3D textiles

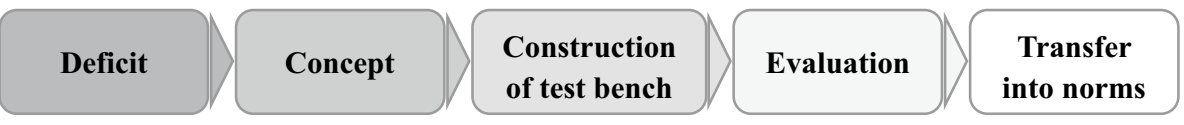

Fig. 2 Procedure during the development of new test methods 
2. development of concepts for the adaptation of existing standardized test methods or for the development of new innovative test methods

3. construction and implementation of the selected concepts

4. validation and iterative improvement of the adapted or newly developed test methods and verification of the industrial suitability of the test devices and regulations

5. transfer/transformation of the findings into new national standards with the involvement of all necessary user groups [cf 2].

This procedure is applied for each of the seven test methods (1. sample preparation, 2. maximum force, 3. thickness 4. compression, 5. mass per unit area, 6. permeability to air, 7. abrasion resistance).

\subsection{Experimental Deficit Analysis}

The following chapter describes the deficits of the existing test methods and test devices of conventional 2D textiles for spacer fabrics. These deficits were determined by testing various spacer fabrics according to the available standards.

\subsubsection{Sample Preparation}

The essential requirements for comparable results are, on the one hand, a uniform definition of terms and, on the other hand, similar samples. For the testing of spacer fabrics, it is necessary to define standardized terms for the components of spacer fabrics and the properties to be tested. Currently, the standard method of sample preparation is punching. During the punching process, a punching iron is pressed through the textile, thus producing consistent sample bodies for conventional 2D textiles. In the case of spacer fabrics, however, the punching process produces tilted samples (Figs. 3 and 4) [3].
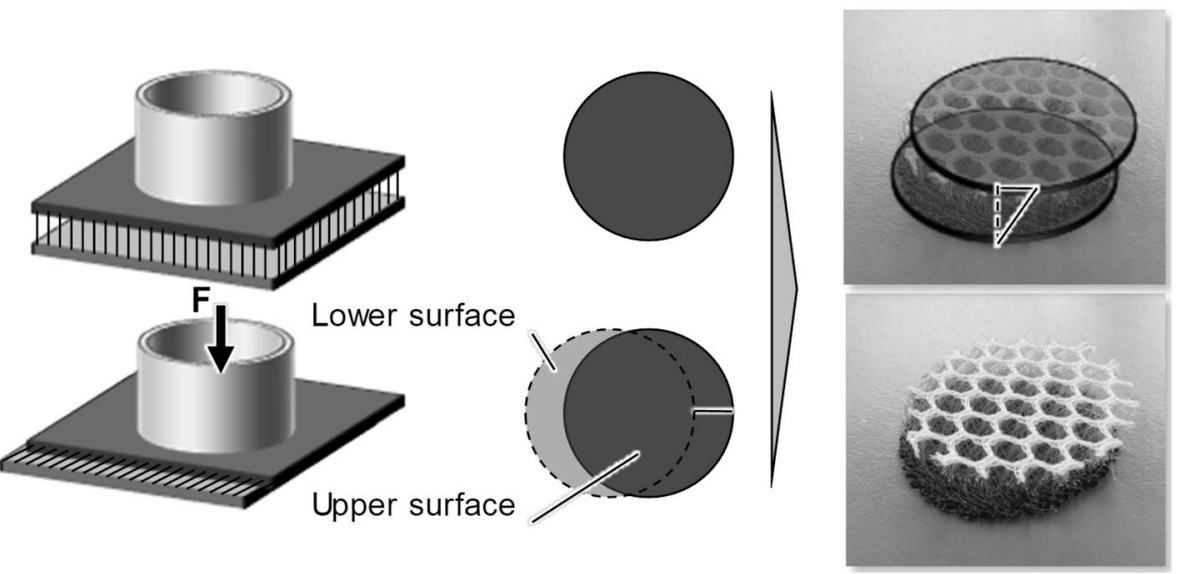

Fig. 3 Schematic illustration of the surface displacement during sample preparation 

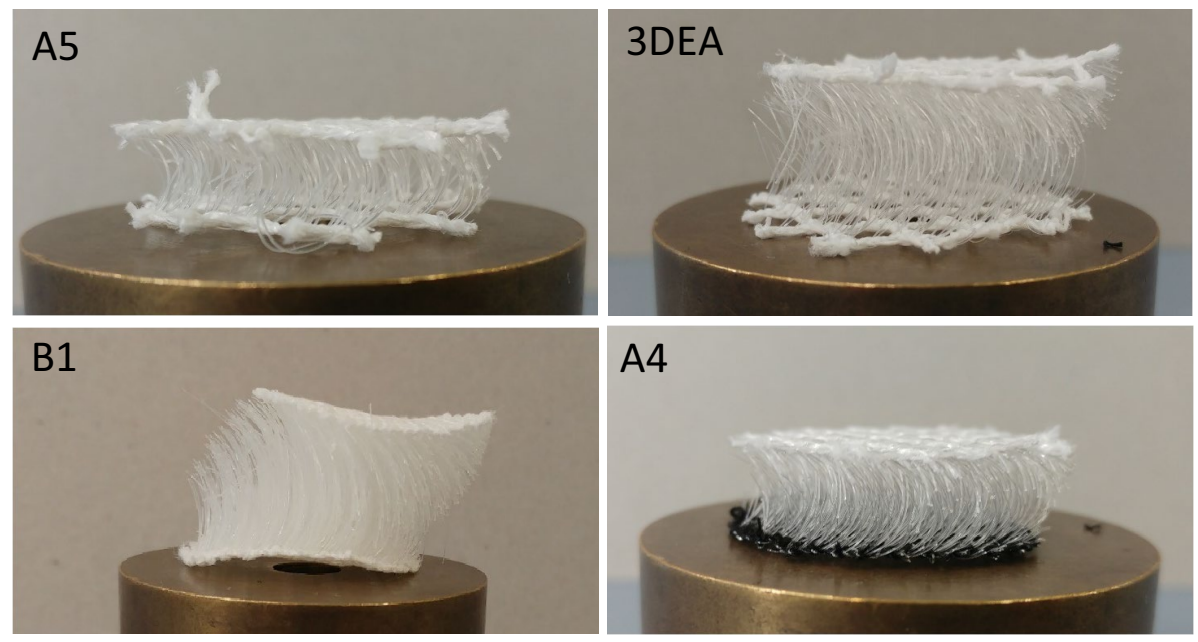

Fig. 4 Surface displacement during sample preparation of spacer fabrics

\subsubsection{Maximum Force}

The current test standard (DIN EN ISO 13934-2: Textiles-Tensile properties of fabricsPart 2: Determination of maximum force using the grab method) for the determination of the maximum force is not suitable for spacer fabrics. Due to the inadequate clamps, clamp breakage occurs. This means, that the textile breaks within the clamping zone due to the high-localised compression between the clamps. Therefore, the maximum force of the textile cannot be detected. It must be noted that this behaviour occurs more frequently the thicker the spacer fabrics is. The thicker the spacer fabrics are, the more these fabrics are stressed at the clamping point.

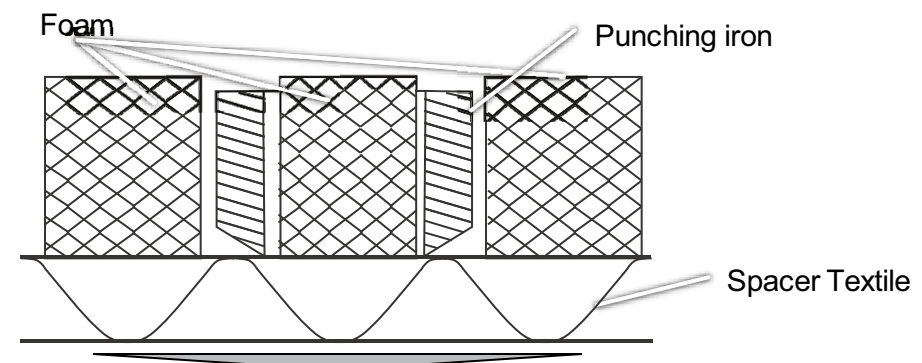

Punch

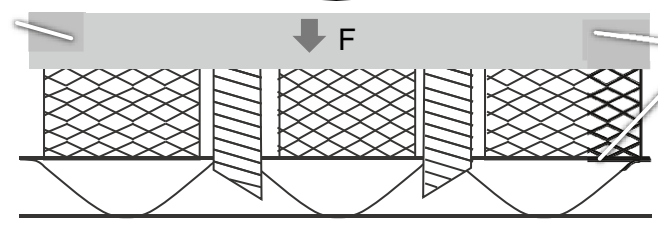

Contact surfaces between punch, foam and textile is expected to prevent slippage

$1 \mathrm{~cm}$

Fig. 5 Concept for sample preparation 


\subsubsection{Thickness}

The standard procedure for the thickness test is not suitable either. By subjecting the spacer fabrics to $1 \mathrm{kPa}$ during the test, the structure is deformed and the actual thickness cannot be measured. Depending on the pile yarn material, different thicknesses are measured for the structures.

\subsubsection{Compression}

The compression testing of textiles is designed for $2 \mathrm{D}$ textiles and not suitable for $3 \mathrm{D}$ fabrics. Shearing of the cover surfaces is not recorded in this test method. The restoring force of the spacer pile yarns is also not considered. Additional the complex deformation behavior is not taken into account.

\subsubsection{Mass Per Unit Area}

There are no test standards for determining the mass per unit area of cover surfaces and pile material. Furthermore, there are deficiencies in the sampling procedure. When the material is punched, the two surfaces are sheared off. Therefore, no exact sample can be taken and the mass per unit area is inaccurate. Consequently, the sample preparation must be taken into account when testing spacer fabrics.

\subsubsection{Permeability to Air}

Measuring the permeability to air currently cannot be done according to the current standard. In spacer fabrics, the air not only flows through the fabric perpendicular to the surface, but can leak out at the sides through the open pile surface plane. The existing protective device is not suitable for spacer fabrics.

\subsubsection{Abrasion Resistance}

The abrasion testing devices are designed for the use of 2D textiles. Spacer fabrics cannot be fixed in the sample grips or press out at the edges during testing. The test conditions are dependent on the mass per unit area, but the thickness is not regarded as important criteria.

\section{Results - Developed Test Methods and Test Benches}

In the following chapter the developed concepts and test benches are described for each of the seven test methods.

\subsection{Sample Preparation}

To conduct comparable tests, it is necessary to use comparable samples. Sample bodies are the result of sample preparation. During the die cutting process, the cover surfaces of spacer fabrics are displaced against each other. To prevent this, a foam is placed between 
the punch and the textile. The foam is slightly compressed and pressed onto the textile surface. The aim is to fix the upper cover surface by friction and prevents it from displacing (Fig. 5).

The evaluated concepts for the sample preparation (e.g. die cutting, laser cutting) are not applicable for all kinds of different spacer fabrics. The variety of spacer fabrics (e.g. thickness, texture, pore size) requires different methods for the extraction of samples for testing. Therefore, the focus of this work is on evaluating the samples produced rather than prescribing how they are produced.

In order to evaluate the result of sample preparation of spacer fabrics, it is necessary to determine the characteristic properties of sample bodies and design suitable evaluation tools. The following Fig. 6 is showing one of the developed tools for the determination of the roundness of a circular sample.

The concepts are designed to define the sample preparation not by the cutting method used, but by their geometric dimensions. This means, that before testing a sample of a material, the suitability of the sample is determined. For this purpose, the decisive characteristics of a sample of a spacer fabric are determined and measuring methods and instruments are designed. Different measuring tools are created for circular and rectangular samples. The tools are used to evaluate roundness, squareness, diameter, edge length and cover offset. Furthermore, concepts for the measurement of the pore/comb/opening size of the cover surfaces of spacer fabrics as well as for the measurement of the pile thread curl are developed and transformed into a standard. The standard "DIN 60022-1 Spacer textiles - Terms and definitions, sample preparation", which has already been published [4], now contains 4 validated test methods for the evaluation of sample quality:

- Roundness/diameter

- Edge length and squareness

- Maximum offset of the top surfaces

- Spacing thread curvature

The following Fig. 7 shows one of the developed tools for evaluating the quality of samples. This angle is used to evaluate the offset of the surfaces occurring due to shearing.

\subsection{Maximum force}

During testing of the maximum force of spacer fabrics, so-called clamp fractures or clamp breakages and invalid tests occur. This means that the sample tears at the clamping
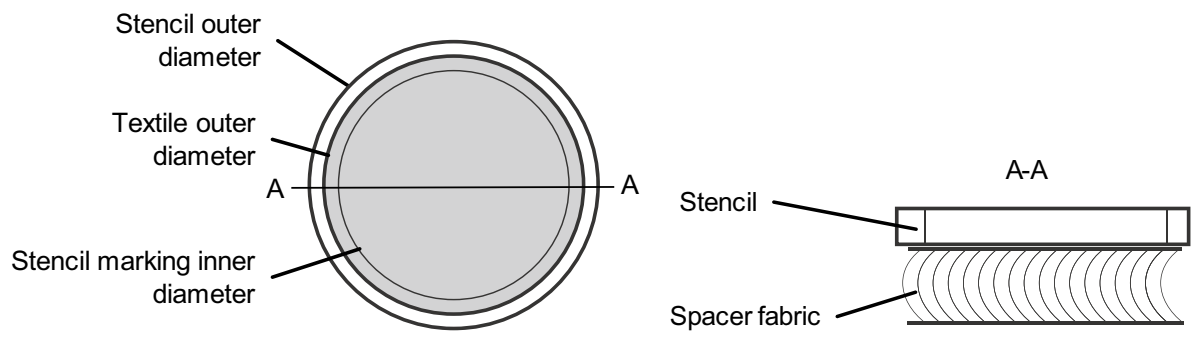

Fig. 6 Concept for the evaluation of sample preparation 
Fig. 7 Measurement of the surface offset s2 (1: angle; 2 : spacer fabric) [5]

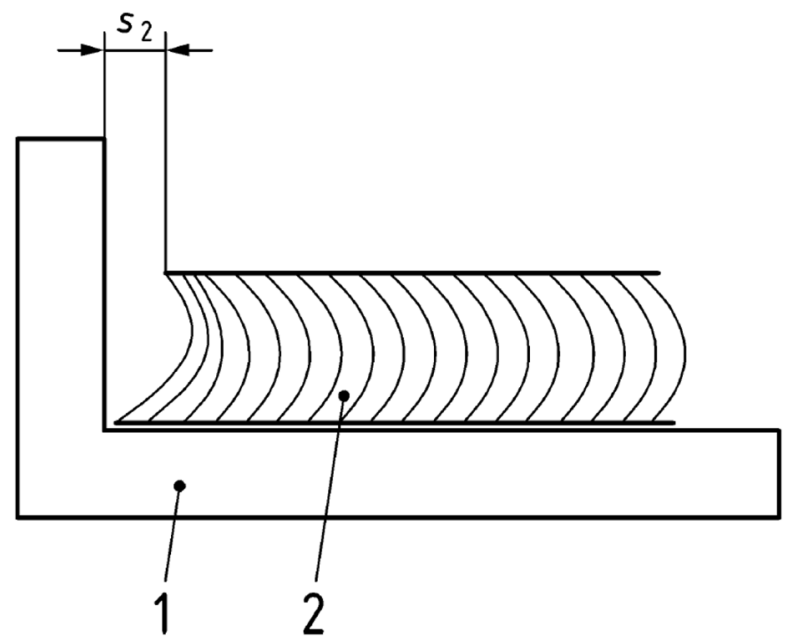

position or "slips" out of the clamp. The clamps are closed with a hydraulic clamp (Type 8293 ) with $10 \mathrm{kN}$ closing force to avoid slipping of the samples within the clamp.

Figure 8 shows the four developed concepts. The aim is to prevent the sample from slipping out by modifying the clamp and to prevent the clamp fractures by modifying

a)

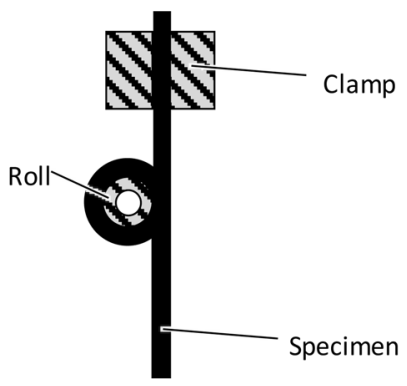

b)

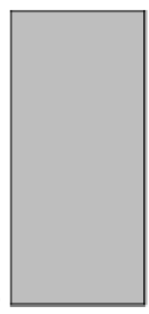

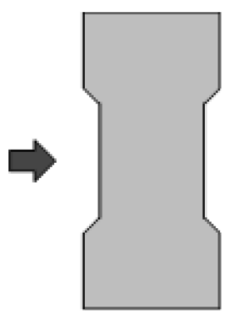

c)
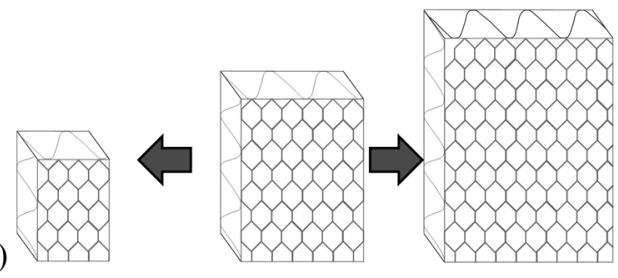

d)

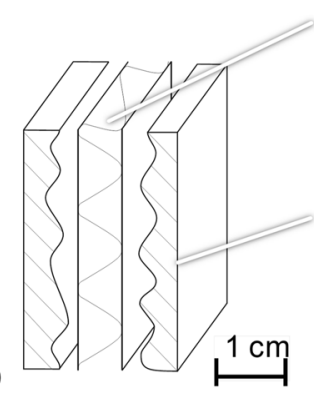

Spacer fabric

Clamp with

waved

pattern

a) Variation of the clamping device b) variation of sample geometry

c) variation of sample size d) clamp with waved pattern

Fig. 8 Concepts for the maximum force test of spacer fabrics 
the sample shape. Different steel and polyurethane clamps with plain and waved surfaces are tested.

It has been shown that none of the three concepts is suitable for testing spacer fabrics when maximum force and elongation are tested in one operation, which is the current standard. Therefore, further research is needed, to determine where the separation of test methods for the determination of maximum force and elongation is possible.

\subsection{Thickness}

In the current test, the operator must add an external weight onto the measuring device before measuring. This can lead to deformation of the textile or to false values, if the weight is not added or if the weight is compressing the spacer fabric. Therefore, the actual thickness cannot be measured. To avoid the deformation, a new test device (Fig. 9) is developed with an incremental weight. Due to the incremental weight the same pressure is always applied to the fabric.

Furthermore, an additional ring was added onto the measuring plate to avoid concave or convex deflection of the samples. To enable the testing of thick spacer fabrics up to $100 \mathrm{~mm}$, the space between measuring plate and measuring stamp was increased.

In summary, the advantages of the new design compared to the Universal Thickness Gauge are listed as follows:

- Larger measuring plate at the bottom-avoids concave or convex deflection of the sample -> This improvement is also applicable for existing measuring devices, as the ring can be added as a spare part.

- Equal contact pressure-avoids compression of the sample

- Measuring unit reacts less sensitive to vibrations

- Measuring range for 3D textiles could be increased from 22 to $100 \mathrm{~mm}$

Fig. 9 Design of the new thickness measuring device with improved setup

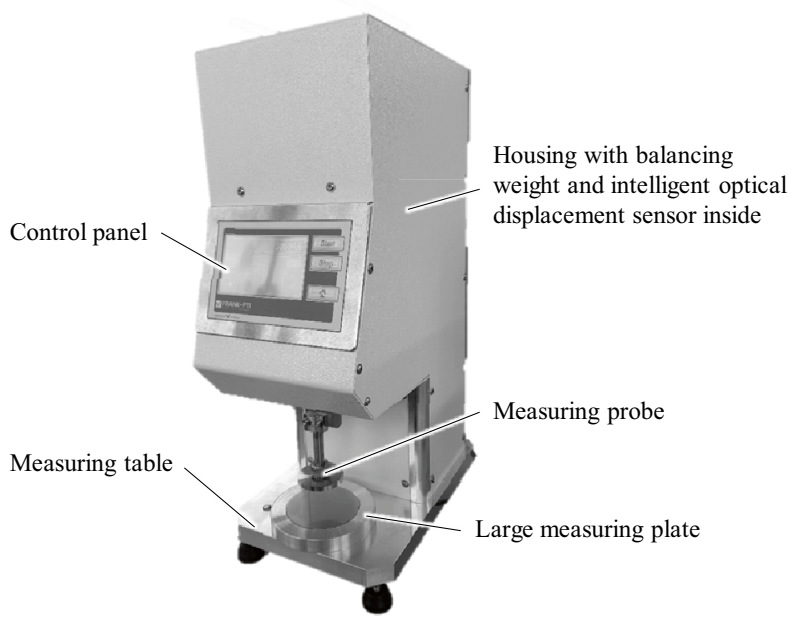


Following the concept development and construction of the test device, the evaluation in comparison to the standard universal thickness tester is performed. Both measuring devices are showing the same results for all kinds of spacer fabrics tested, but the reproducibility (spreading of values) was better with the new device. The new method is currently transformed into a national standard.

\subsection{Compression}

In compression testing, according to the current standard, a textile is loaded with two different forces and the corresponding compression is measured. Complex behavior of the material during compression is not taken into account. In addition, no restoring force is determined.

In Fig. 10 an example for a graph of the compression test is shown. The comparison of the force-elongation curve when testing spacer textiles with $40 \%$ and $70 \%$ compression is presented, each with $1 \mathrm{~min}$ holding time and speed $10 \mathrm{~mm} / \mathrm{min}$.

The samples compressed to $40 \%$ have no significant differences between the compression curves of the first cycle and the curves of the subsequent cycles (red curves). The curves are congruent. The conclusion is, that the compression to $40 \%$ of the original thickness of the sample does not damage the sample. The violet curves, on the other hand, are not congruent. The compression behaviour of the sample changes over the number of cycles. This leads to the conclusion, that the sample is damaged during the compression test.

In the following, further test series are carried out to find a suitable testing procedure for spacer fabrics without damaging the material. The following parameters were varied:

- Properties of spacer fabrics (different thicknesses, different pile thread densities, open and closed cover areas),

- test speed,

- compression value,

- holding time and

- stamp shape and size used.

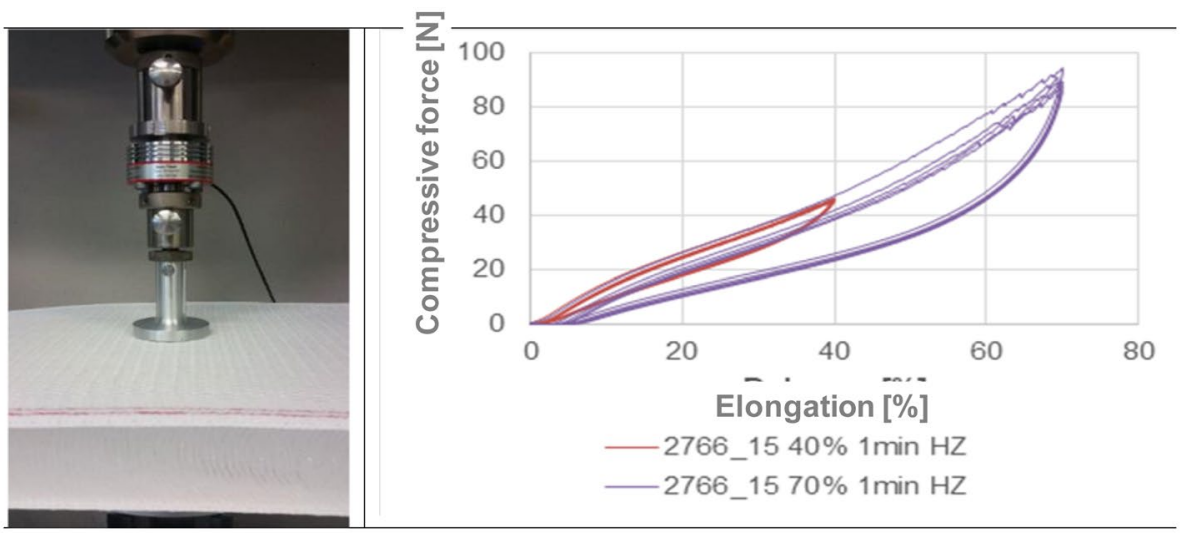

Fig. 10 Comparison of the force-elongation curve when testing spacer fabrics with $40 \%$ (red) and $70 \%$ (violet) compression, $1 \mathrm{~min}$ holding time in each case 
The results of the compression tests and the adaptation of the methods to spacer fabrics are still showing deficits. Therefore, further research is needed with a new approach, where the pressure curve is analyzed regarding characteristic points to describe the compression properties of spacer fabric without pre-damaging the textiles.

Consequently, Fig. 11 shows a concept for a compression test on spacer fabrics. The spacer fabric is compressed in a compression testing machine and the force-displacement curve is determined and recorded. Characteristic points in the measurement chart can be determined and provided as output. This test is also taking into account the complex behavior of the material during compression.

\subsection{Mass Per Unit Area}

The areal weight of spacer fabrics is directly dependent on the quality of sample preparation. Compared to the testing of 2D textiles, however, there are no deficiencies in the testing of spacer fabrics. Therefore, the consortium decides to consider sample preparation as an important process in the testing of spacer fabrics and to present it to the standards committee. The standards committee decides to work on the standardization of sample preparation instead of the standard for the determination of the mass per unit area.

\subsection{Permeability to Air}

For spacer fabrics, in addition to the conventional flow direction through the cover surfaces, another flow direction is of interest: the air flow through the spacer threads, meaning in the horizontal direction. Moreover, the clamping bell of the standardized test rig for air permeability testing is not suitable for clamping spacer fabrics, since the lateral leakage of the test air cannot be sealed. Figure 12 shows the test concepts for testing air permeability in the horizontal and vertical directions. For vertical testing (Fig. 12a) the sides of the sample are sealed to prevent leakage. For the horizontal test (Fig. 12b), the top surfaces of the spacer fabric are sealed.

Figure 13 shows the test setup for testing horizontal air permeability. The test sample is placed and fixed between two movable barriers. The barriers can be adjusted to the thickness of the spacer fabric. The rest of the test setup remains unchanged.

Fig. 11 Concept for compression test

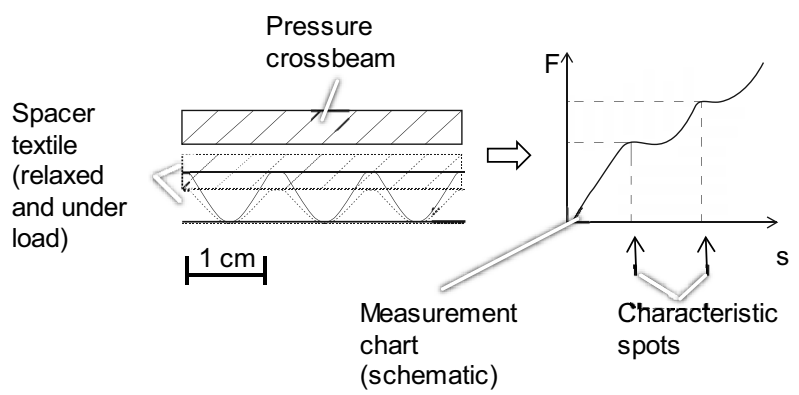



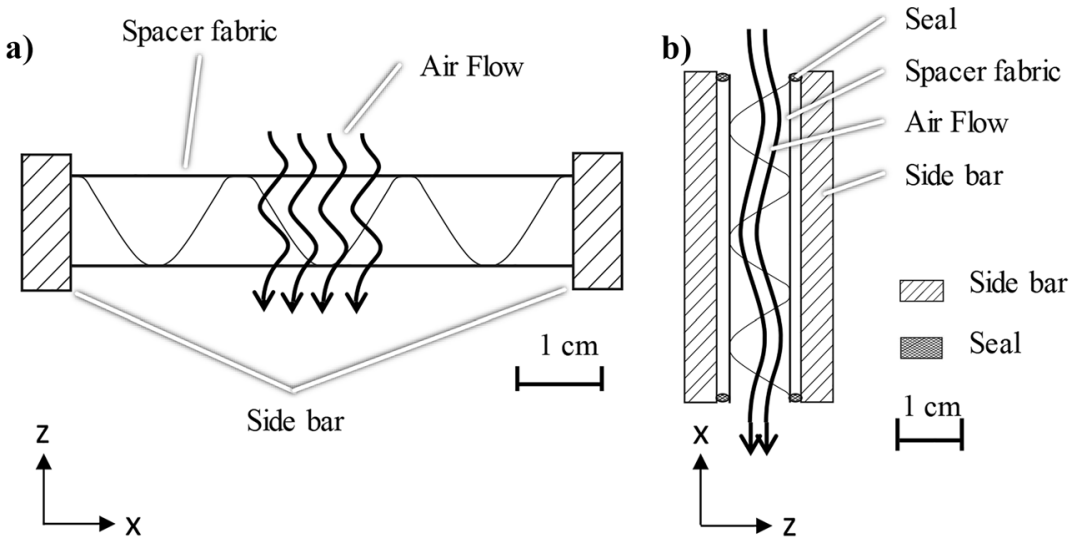

a) vertical air flow through the cover surfaces, b) horizontal air flow through the spacer threads

Fig. 12 Concept for permeability to air test

Figure 14 shows the test setup for testing vertical air permeability. The triangle is used to position the test attachment on the test instrument (here FX 3300 LabAir, TEXTEST AG, Switzerland).

Following the concept development and construction of the test devices, the evaluation in comparison to standard measuring devices is performed and the method is currently transformed into a national standard.

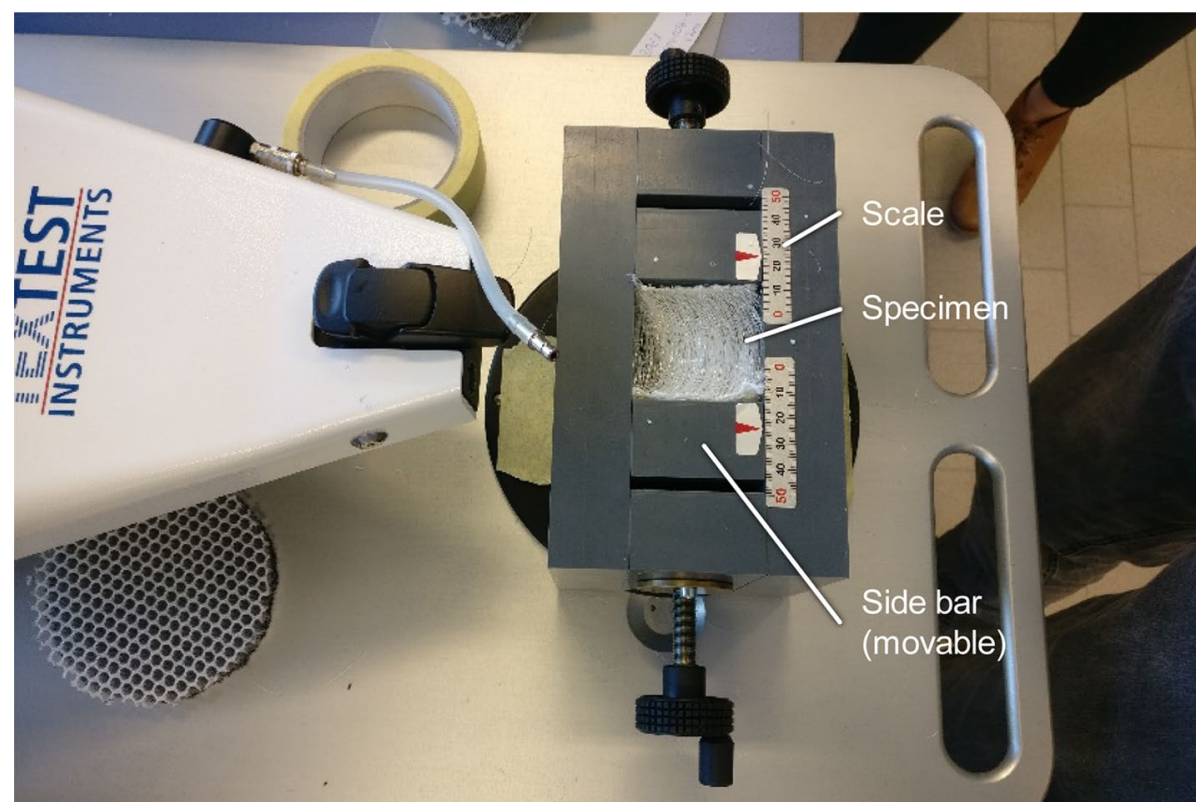

Fig. 13 Test bench for permeability to air test 


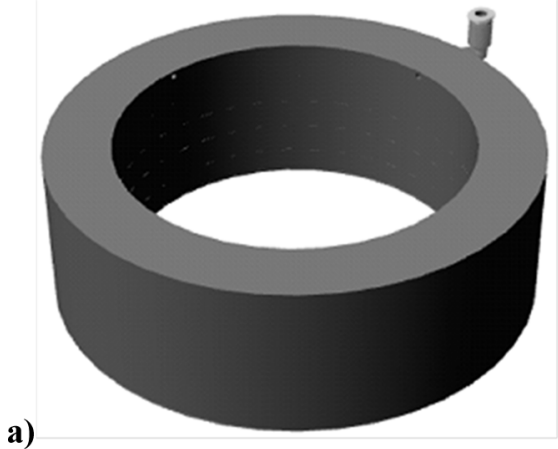

a) design b) implementation

b)

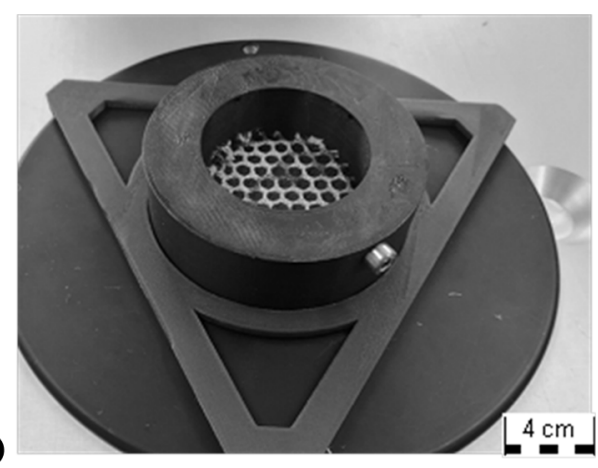

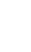

Fig. 14 Setup of the vertical air permeability test

\subsection{Abrasion Resistance}

In the abrasion test, it is currently not possible to place very thick textiles, such as spacer fabrics, in the sample holder. Therefore, in the concept shown in Fig. 15, the sample holder is changed. Instead of the usual closed sample holder, an open system is proposed. The textile sample is placed and fixed around the sample holder.

Figure 16 shows the test bench for the abrasion test. On the left is the newly designed sample holder, which was manufactured using a 3D printer. On the right, the assembled test setup is shown. The sample is mounted using a rubber band. The rest of the test setup remains unchanged.

In addition to the concept of a new sample holder, the size of the samples is varied and tested with a standard sample holder. Further research is necessary to finalize the validation phase and transfer the results into standards.

Fig. 15 Concept for abrasion resistance test

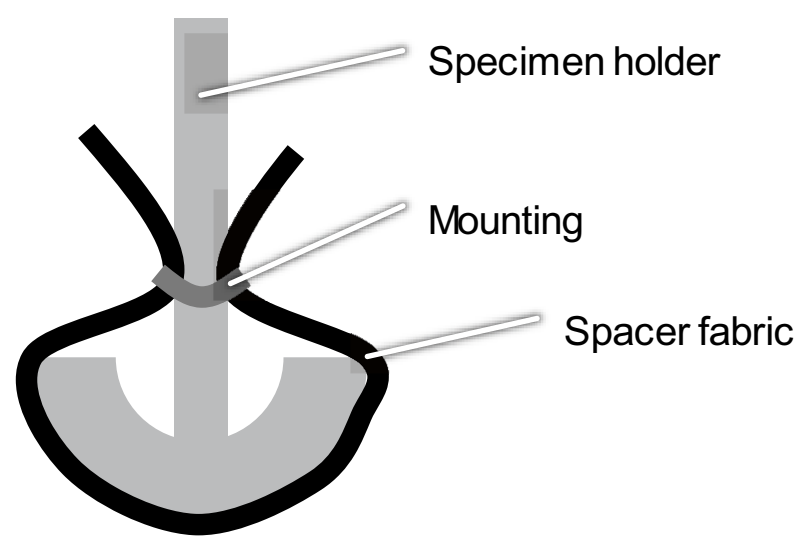


Fig. 16 Test bench for abrasion resistance test

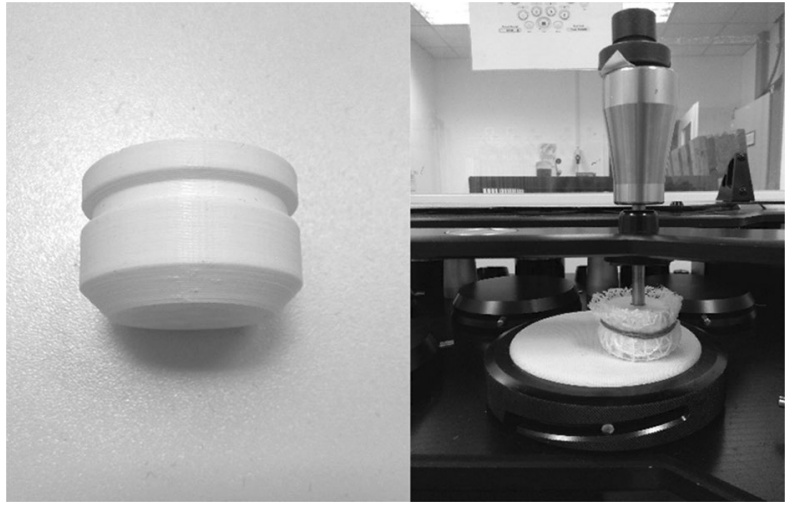

\section{Discussion}

Within this research, seven tests were examined with regard to their applicability to spacer fabrics. For this purpose, the available standards were examined and their deficits in application to spacer fabrics were determined. Subsequently, concepts for the adaptation of standards for $2 \mathrm{D}$ textiles as well as completely new standards were developed. The subsequently constructed and manufactured test benches were then evaluated in the industrial environment.

It was shown that the biggest deficit is the unclear definition of spacer fabrics and their characteristics as well as the variety of materials to be tested. Therefore, several concepts for the uniform evaluation of cut samples were developed and transferred to a standard, together with definitions of terms for spacer fabrics. This newly developed standard for the sample preparation is also an important basis for the determination of the mass per unit area, as sample preparation was the only deficit of the determination of the mass per

Table 1 Test methods for textiles and deficit for spacer fabrics

\begin{tabular}{|c|c|c|}
\hline Test & Standard & Deficits for the testing of spacer fabrics \\
\hline Sample preparation & No standard available & $\begin{array}{l}\text { - Definition of terms is not available } \\
\text { - Standard sample preparation of spacer fabrics can lead to } \\
\text { false results }\end{array}$ \\
\hline Maximum Force & $\begin{array}{l}\text { DIN EN ISO 13,934-2 } \\
\mathbf{2 0 1 4}\end{array}$ & - Clamps are not suitable and clamp breakage occurs \\
\hline Thickness & $\begin{array}{l}\text { DIN EN ISO } 5084 \\
\mathbf{1 9 9 6} \text { [6] }\end{array}$ & - Loading of the textile with weight falsifies the test result \\
\hline Compression & $\begin{array}{l}\text { DIN 53,885 } \\
\mathbf{1 9 9 8}[7]\end{array}$ & $\begin{array}{l}\text { - Shearing of the cover surfaces } \\
\text { - Restoring force } \\
\text { - Complex deformation behavior are not taken into account }\end{array}$ \\
\hline Mass per unit area & $\begin{array}{l}\text { DIN EN 12,127 } \\
\mathbf{1 9 9 7}[8]\end{array}$ & - Shearing of the surfaces during sample preparation \\
\hline Permeability to air & $\begin{array}{l}\text { DIN EN ISO } 9237 \\
\mathbf{1 9 9 5} \text { [9] }\end{array}$ & - Air can leak out at the sides \\
\hline Abrasion resistance & $\begin{array}{l}\text { DIN EN ISO 12,947 } \\
\mathbf{2 0 0 7}[10]\end{array}$ & $\begin{array}{l}\text { - Test conditions depend on mass per unit area } \\
\text { - Pressing out of sample grips } \\
\text { - No clamping of thick samples possible }\end{array}$ \\
\hline
\end{tabular}


unit area for spacer fabrics. The newly developed standard for the sample preparation was already published and is already used.

The results of the maximum force test methods and the adaptation of the methods to spacer fabrics are still showing deficits. According to the state of the art, the maximum force and the elongation were tested in one procedure. This leads to restrictions in the adaptation of the test methods to spacer fabrics, e.g. the sample dimension. Therefore, further research is needed with a new approach, where separate methods for the determination of maximum force and elongation are developed.

This work is showing, that with the newly developed thickness measuring gauge for spacer fabrics the results are reproducible. The new design of the measurement device avoids erroneous results due to pre-damage of the textile and due to curved support of the textile. The developed standard draft is currently under review.

The results of the compression tests and the adaptation of the methods to spacer fabrics are still showing deficits. Therefore, further research is needed with a new approach, where the pressure curve is analyzed regarding characteristic points to describe the compression properties of spacer fabric without pre-damaging the textiles.

For the determination of the permeability to air it was shown, that both test directions (horizontal and vertical) are showing reproducible results. Therefore, the developed concepts were transferred into a standard draft after evaluation. The developed standard draft is currently under review.

The developed concepts as well as the new specimen holder for the test of the abrasion resistance are showing good results within this research. Nevertheless, the evaluation on an industrial scale could not yet be completed. Therefore, further research is needed.

\section{Conclusions and Outlook}

Within the presented research, the deficits of testing spacer fabrics were analyzed, concepts and test benches were developed, evaluated and successfully transformed into one published standard document and two standard drafts. The interdisciplinary cooperation during the conducted research between manufacturers of spacer fabrics, test equipment manufacturers, construction experts, textile research institutes and test service providers as well as the German Institute for Standardization was of central importance.

The newly developed standard "DIN 60022-1 Spacer textiles - Terms and definitions, sample preparation" [4] was published in 2020 and is now available. It is already used by the textile industry. Two further standards drafts were developed: thickness measurement and permeability to air. Both standards are currently being reviewed and will be published for public comment within 2021.

In addition to the test methods developed within this work, further research is necessary. In particular, the in-plane and out-of-plane shear measurement as well as the compression behaviour of spacer fabrics are important tests that need to be analysed and further developed. Therefore, further research is planned for six test methods (1. Compression hardness, 2. Compression set, 3. Tilting stability, 4. Pressure point distribution, 5. Abrasion resistance, 6. Maximum force).

Furthermore, since these results are mostly developed to fit the requirements of warp knitted spacer fabrics, the research results will be evaluated regarding weft knitted and woven spacer textiles afterwards. This work enables standardized testing of spacer fabrics 
and thus objective comparisons not only between various spacer fabric constructions but also with conventional flat textiles and with non-textile materials.

Acknowledgements The authors would like to thank the Federal Ministry of Economics and Technology for supporting this research under the WIPANO program "Wissens- und Technologietransfer durch Patente und Normen".

The research was accompanied by a user-related technical committee and a standards committee for the development of the standardisation. The authors would also like to thank the various companies involved for their contribution.

The research project "NormATex" is primarily supported by the German Institute for Standardization (DIN e.V.), Berlin, who is responsible for converting the developed testing methods into standards and norms. The Institut für Textiltechnik (ITA) of RWTH Aachen University, Aachen, Frank PTI GmbH, Birkenau, HK Konstruktion, Weinheim, and $\mathrm{t}+\mathrm{h}$ ingema Ingenieurgesellschaft $\mathrm{mbH}$, Aachen, are working towards the development of new testing methods.

Funding Open Access funding enabled and organized by Projekt DEAL. The project is funded under the WIPANO programme-"Wissens- und Technologietransfer durch Patente und Normen" of the Federal Ministry of Economics and Technology (BMWi).

Data Availability, Material, and Code The data, material and code can be found at the following contacts: Carolin Schwager, email: carolin.schwager@ita.rwth-aachen.de

\section{Declarations}

\section{Conflicts of Interest/Competing Interests None}

Open Access This article is licensed under a Creative Commons Attribution 4.0 International License, which permits use, sharing, adaptation, distribution and reproduction in any medium or format, as long as you give appropriate credit to the original author(s) and the source, provide a link to the Creative Commons licence, and indicate if changes were made. The images or other third party material in this article are included in the article's Creative Commons licence, unless indicated otherwise in a credit line to the material. If material is not included in the article's Creative Commons licence and your intended use is not permitted by statutory regulation or exceeds the permitted use, you will need to obtain permission directly from the copyright holder. To view a copy of this licence, visit http://creativecommons.org/licenses/by/4.0/.

\section{References}

1. German Association of the Automotive Industry (VDA), Berlin, Germany https://www.vda.de/de/ services/zahlen-und-daten/jahreszahlen/allgemeines.html. Access: 21.11 (2016)

2. Standardization - Part 4: Working procedure; German version DIN 820-4:2021-02, Berlin, Beuth (2021)

3. Peiner, C., Bettermann, I., Blaurock, C., Lehn, R., Rusinski, T., Kampfl, H., Abu Namous, D.: Normung für Abstandstextilien, Project final report, Technische Informationsbibliothek (TIB), Hannover, Germany (2020)

4. DIN 60022-1 Spacer textiles - Terms and definitions, sample preparation, Berlin, Beuth (2020)

5. Textiles - Tensile properties of fabrics - Part 2: Determination of maximum force using the grab method (ISO 13934-2:2014); German version EN ISO 13934-2:2014, Berlin, Beuth (2014)

6. Textiles - Determination of thickness of textiles and textile products (ISO 5084:1996); German version EN ISO 5084:1996, Berlin, Beuth (1996)

7. Textiles - Determination of compression of textiles and textile products; German version DIN 53885:1998-12, Berlin, Beuth (1998)

8. Textiles - Fabrics - Determination of mass per unit area using small samples; German version EN 12127:1997, Berlin, Beuth (1997)

9. Textiles - Determination of permeability of fabrics to air (ISO 9237:1995); German version EN ISO 9237:1995, Berlin, Beuth (1995) 
10. Textiles - Determination of the abrasion resistance of fabrics by the Martindale method - Part 2: Determination of specimen breakdown (ISO 12947-2:2007); German version EN ISO 12947-2:2007, Berlin, Beuth (2007)

Publisher's Note Springer Nature remains neutral with regard to jurisdictional claims in published maps and institutional affiliations.

\section{Authors and Affiliations}

\section{Carolin Schwager $^{1} \cdot$ Christoph Peiner $^{1} \cdot$ Isa Bettermann ${ }^{1} \cdot$ Thomas Gries $^{1}$}

\section{Carolin Schwager}

carolin.schwager@ita.rwth-aachen.de

1 Institut Für Textiltechnik of RWTH Aachen University (ITA), Aachen, Germany 